\title{
Immunostimulatory and growth promoting effects of sugarcane (Saccharum officinarum L.) extracts in industrial broiler chickens
}

\author{
Masood Akhtar; M.M. Awais, Faqir Muhammad ${ }^{1}$, Iftikhar Hussain ${ }^{2}$, \\ Ahsan Ul Haq ${ }^{3}$ and M. Irfan Anwar \\ Immunoparasitology Laboratory, Department of Parasitology \\ 1- Department of Physiology and Pharmacology \\ 2- Department of Microbiology \\ 3- Department of Poultry Science \\ University of Agriculture, Faisalabad-38040, Pakistan
}

\begin{abstract}
Aqueous extract from sugar cane (Saccharum officinarum) juice and ethanolic extract from bagasse were used in the present study to determine the antibody response to sheep red blood cells and lymphoproliferative response to Phytohemagglutinin-P (PHA-P) in experimental chickens as compared to control. Results revealed significantly higher $(\mathrm{P}<0.05)$ lymphopoproliferative responses in experimental chickens as compared to control at 24, 48 and 72 hours post PHA-P injection. Further, significantly higher $(\mathrm{P}<0.05)$ lymphoproliferative responses were detected in chickens administered with ethanolic extract as compared to chickens administered with aqueous extract. Total, IgG and IgM anti-SRBC antibody titers were significantly higher $(\mathrm{P}<0.05)$ in ethanolic extract administered chickens as compared to aqueous extract administered chickens. The organ body weight ratio of the lymphoid organs of experimental and control groups were statistically nonsignificant $(\mathrm{P}>0.01)$. Chickens of both the experimental groups showed better FCR and significantly higher $(\mathrm{P}<0.05)$ weight gains as compared to control. It was concluded that oral administration of sugar cane extracts showed immunostimulatory effects in chickens and resulted in the improvement of food utilization and decrease in the amount of food needed for unit gain in body weight.
\end{abstract}

